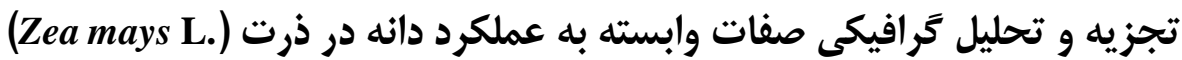

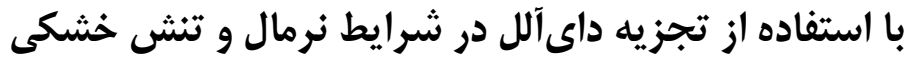

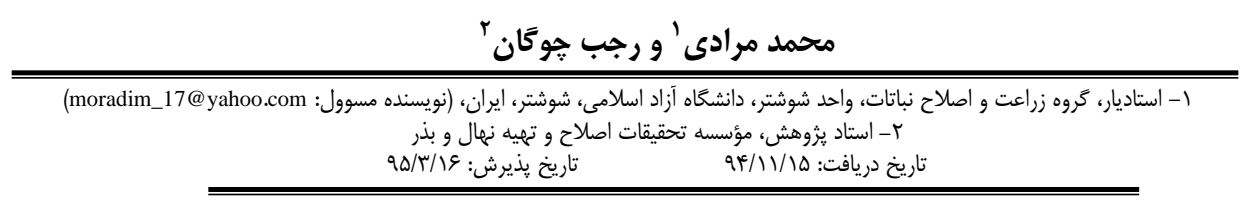

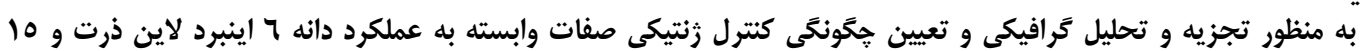

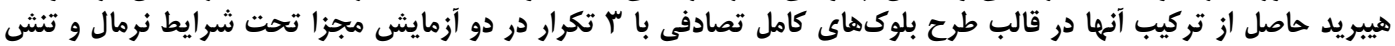

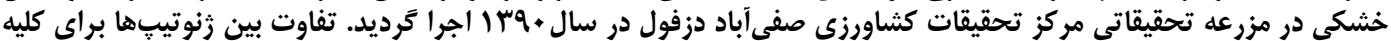

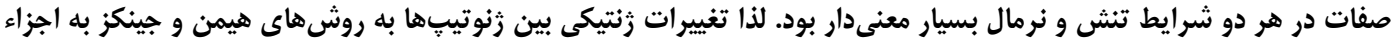

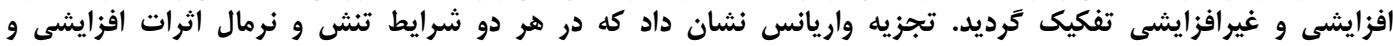

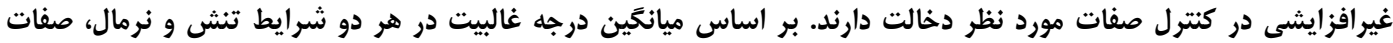

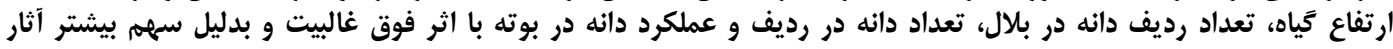

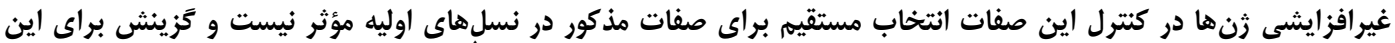

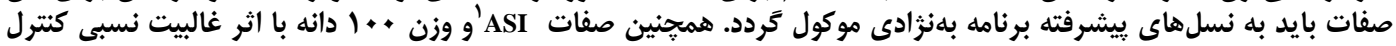

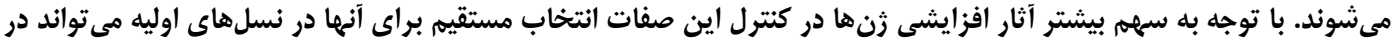
هر دو محيط نرمال و تنش مفيد باشد.

وازههاى كليدى: ذرت، تجزيه و تحليل تَرافيكى، يارامترهاى زنتيكى، خشكى

اطلاعات زنتيكى در خصوص توزيع آللها، ميانخين درجه

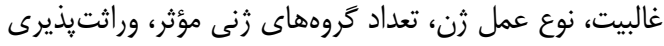

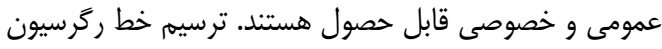

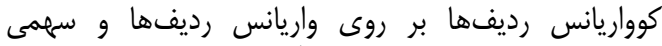

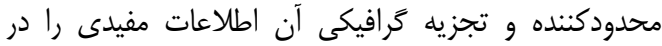

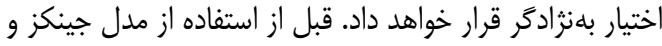

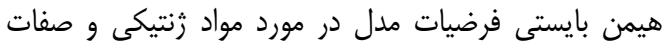

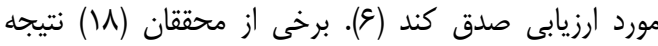

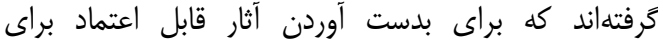

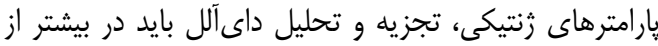

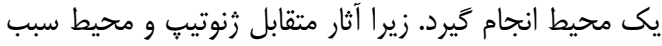

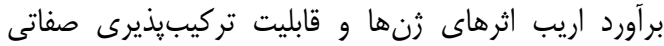

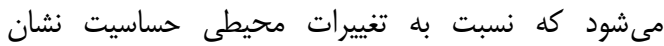

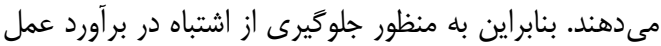

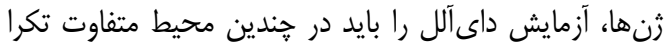

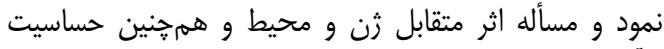

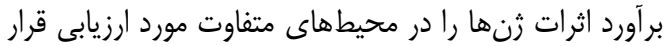

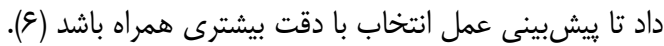

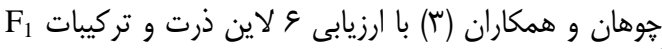

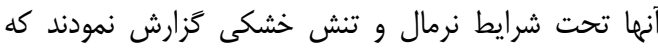

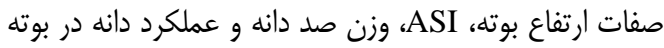

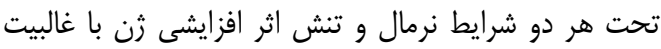

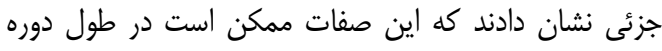

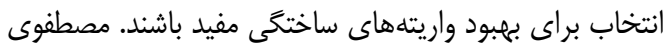

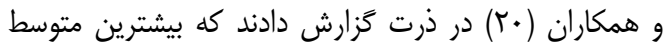

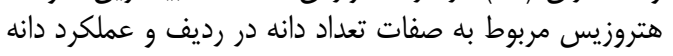
بود. نسبت واريانس افزايشى به غالبيت نشان داد كه در كنترل

1- Anthesis Silking Interval
ذرت (Zea mays L.) از غلات مهمه و با ارزش مناطق

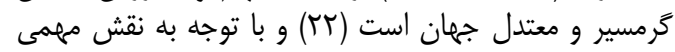

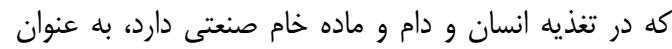

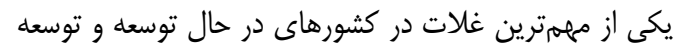

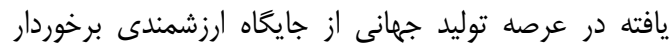

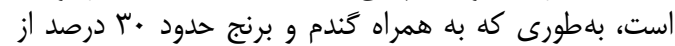

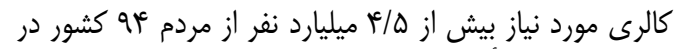

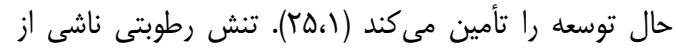

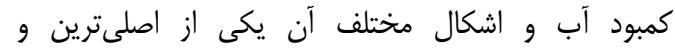

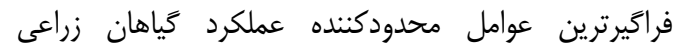

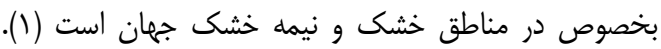

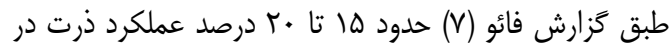

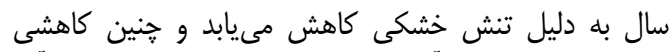

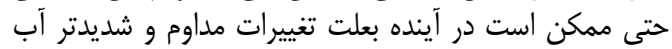

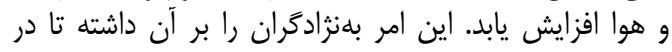

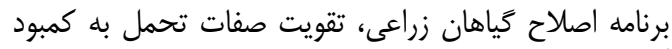

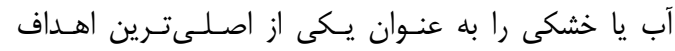

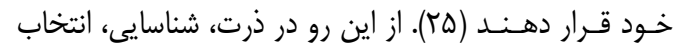

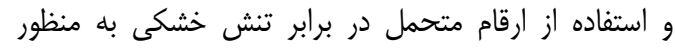

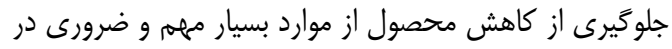

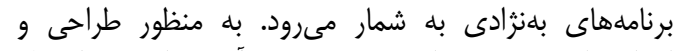

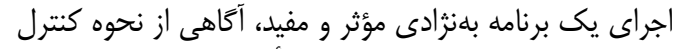

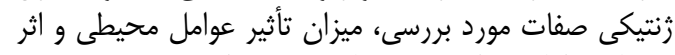

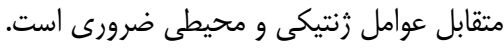

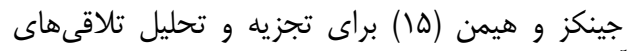
داى آلل روشى را مطرح كردند كه از طريق ائه اين روش تلافي 


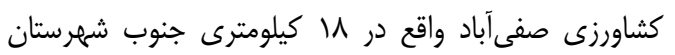

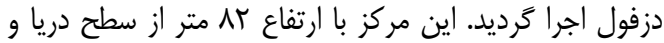

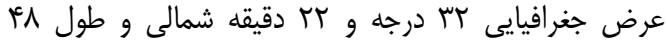

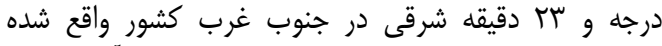

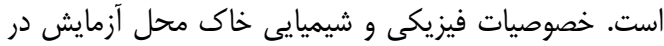

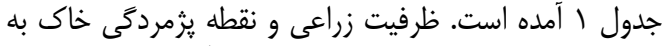

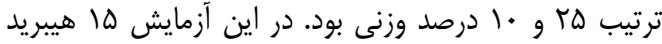

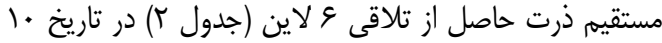

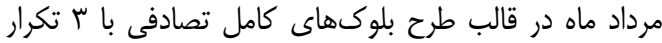

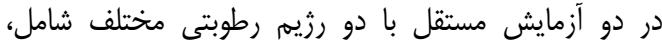

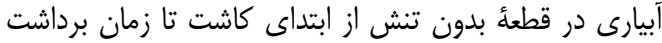

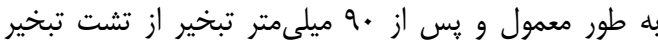

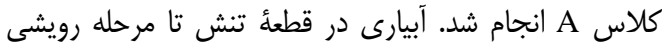
بردا

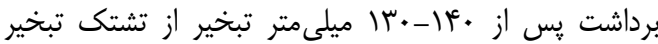

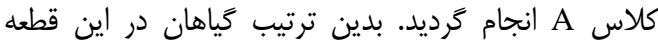

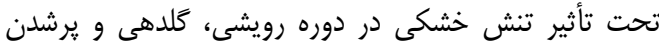

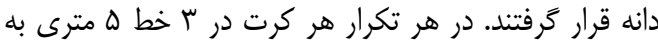

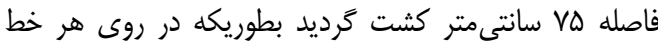

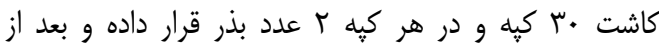

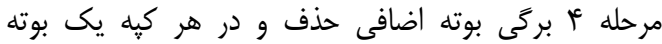

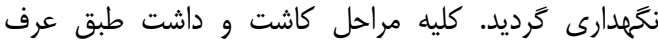

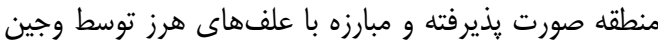

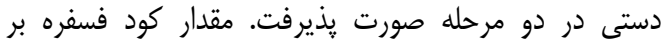

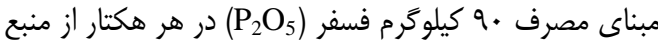

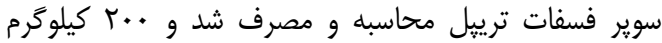

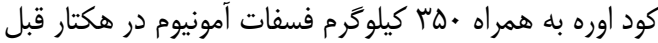

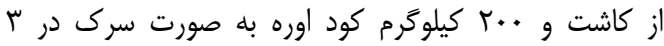
نوبت به مقدار مساوى به فاسله هر يك ماه ماه در هر آز آزمايش

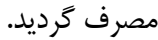

صفات تعداد رديف دانه در بلال و وزن صد دان دانه نقش اثر ائر

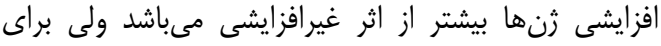

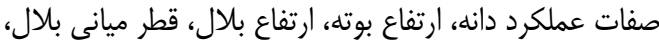

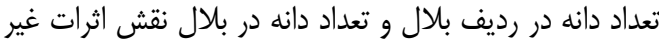

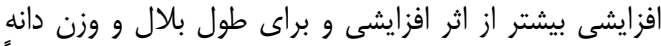

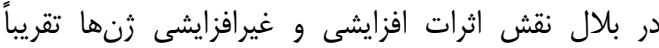

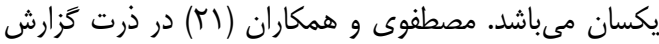

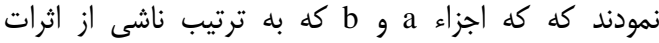

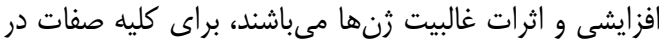

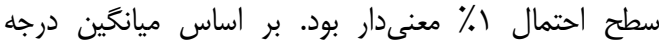

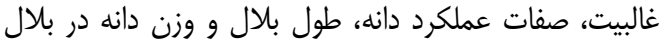

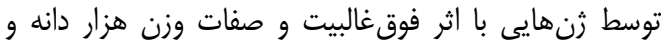

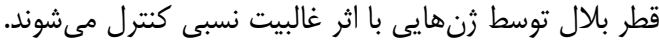

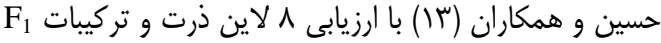

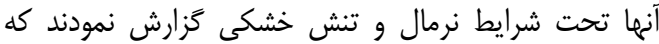

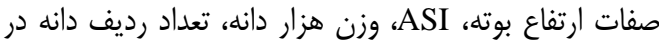

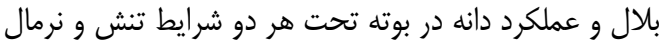
توسط اثرات فوق غالبيت زنها كنترل منى شئه

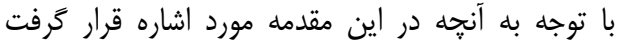

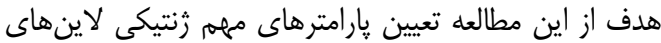

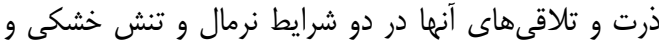

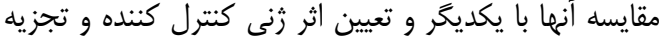

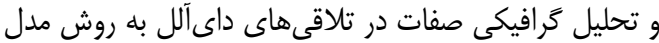

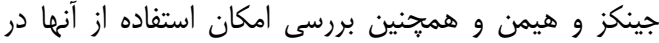

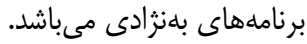

\section{مواد و روشها}

اين يثوهش در سال زراعى |وس||-•وسا| در دو محيط

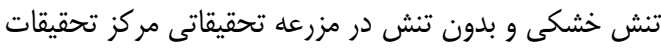

Table 1.Physical and chemical properties of the test site

جدول 1- خصوصيات فيزيكى و شيميايى خاك محل آزمايش

\begin{tabular}{|c|c|c|c|c|c|c|c|}
\hline 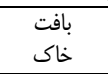 & $\begin{array}{l}\text { يتاسيم } \\
\text { P.P.M }\end{array}$ & $\begin{array}{c}\text { ف.P.M } \\
\text { P.P.M }\end{array}$ & $\begin{array}{c}\text { نيتروثن ك.P.M } \\
\text { P.M }\end{array}$ & أشباع (PH) إيته & هيلى مدايت الكتر بكى برحسترب & كربن آلى & $\begin{array}{c}\text { عمق نمونهبردارى (سانتى) } \\
\text { (سمترى }\end{array}$ \\
\hline رسى-لومى & $|T|$ & $9 / \Delta$ & $r \Delta / \varepsilon$ & $V / r$ & $1 / F T$ & .199 & $\cdot-r \cdot$ \\
\hline رسى-لومى & 110 & Q/4 & $18 / 1$ & $V / \Delta$ & $\cdot / v \Delta$ & .198 & $r \cdot-9$. \\
\hline
\end{tabular}

Table 2. List, Code and Origin Inbred lines used in test

جدول ז- فرمول لاين، نام اختصارى و منشاء اوليه لاينهاى مورد بررسى

\begin{tabular}{|c|c|c|c|}
\hline رديف & فرمول لاين & نام اختصارى لاين & منشاء لاين \\
\hline 1 & CML384 & CML & سيميت(CIMMYT) \\
\hline r & $S D \backslash(3-6) \backslash 2-2-1-2-m-1$ & $\mathrm{SD} 13$ & مركز تحقيقات كشاورزى صفى آباد \\
\hline$r$ & SD\172-1-2-2-2-1-1 & SD\17 & مركز تحقيقات كشاورزى صفىآباد \\
\hline f & SD\10\1-1-1-1-3-1-1 & SD $\backslash 10$ & مركز تحقيقات كشاورزى \\
\hline$\Delta$ & SD\15\1-1-1-2-1-1-1 & SD\15 & مركز تحقيقات كشاورزى ـ \\
\hline 8 & SDI704\4-1-1-3-1-1 & SDI704 & مركز تحقيقات كشاورزى صفى آباد \\
\hline
\end{tabular}

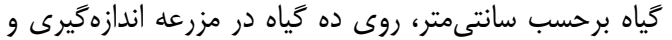

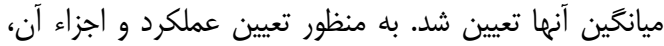

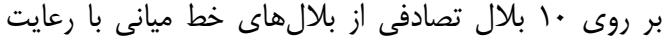

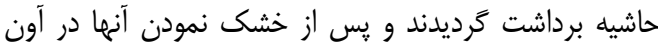

به منظور بررسى صفات از هر كرت آزمايشى خط اول و

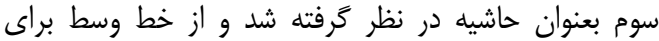

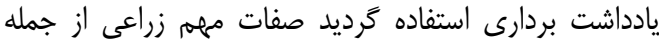

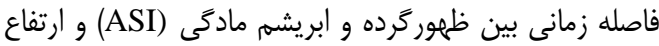




\section{نتايج و بحث} تفاوت بين رنوتيبها براى كليه صفات در دو محيط تنش بنا

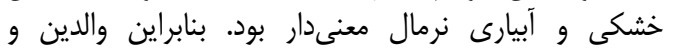

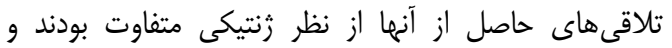

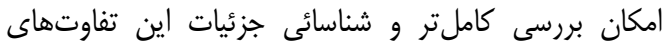

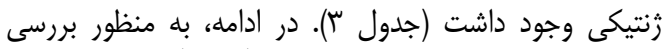

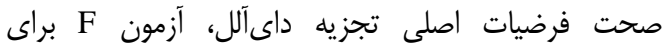

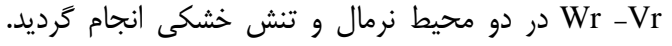

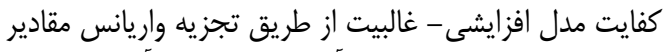

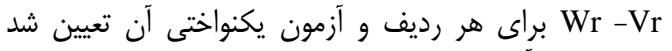

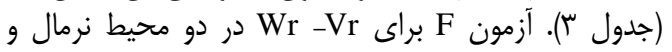

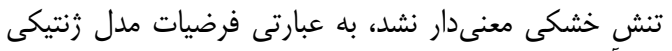

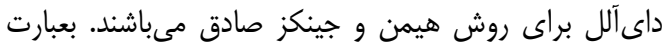

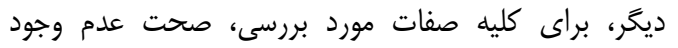

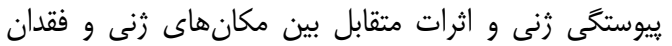

$$
\text { آللها هندكانه برقرار است. }
$$

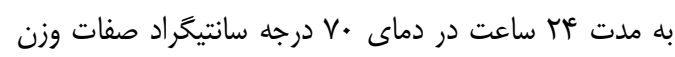

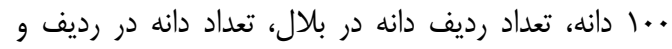

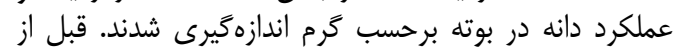

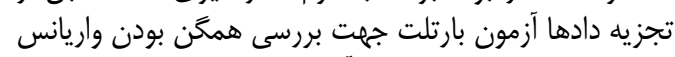

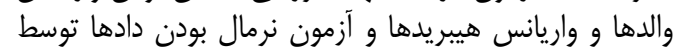

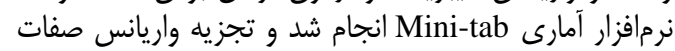
مختلف در قالب طرح بلوكهاى كامل إنل تصادفى، مقايسه

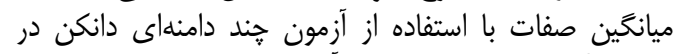

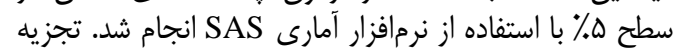

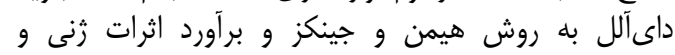

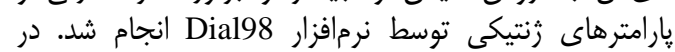

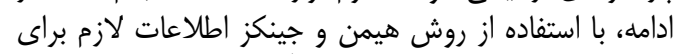

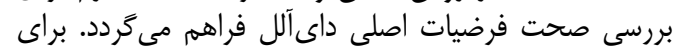

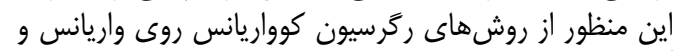

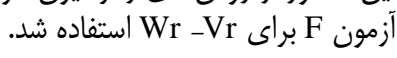

جدول سـ- تجزيه واريانس مقادير Wr _Wr در صفات مختلف ذرت براى كفايت مدل افزايشى- غالبيت در شرايط نرمال و تنش خشكى Table 3. Scaling tests for adequacy of additive-dominance model for various traits in maize under normal and water

\begin{tabular}{|c|c|c|c|c|c|c|c|c|}
\hline عر بوتكرد دانهم) & رديف بلانه در & تعداد رديف دانه & 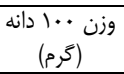 & ASI & (سارتنىمتر) & شرايط & آزازدى & تغنيير \\
\hline$r r V / T$ & $9 / \vee \wedge$ & $1 / \mathscr{\mu}{ }^{\prime \prime}$ & $1 . / 1 \mathbb{S}^{\prime \prime}$ & 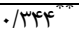 & $V E N / I^{\prime \prime}$ & نومال & & \\
\hline $\mid T N / \Lambda^{\pi *}$ & $11 / v \Lambda^{*}$ & $r / \mathcal{N} \mathcal{E}^{\circ}$ & $\mathrm{V} / \mathrm{AV}$ & "זr.|. & $8 \Delta r / \Lambda^{\mathrm{ns}}$ & تنش & r & تكرار \\
\hline$r \Delta \Lambda / v^{n s}$ & $1 / v^{n s}$ & $\cdot / \mu^{\mathrm{ns}}$ & $r / v q^{n s}$ & $\cdot / \cdot \varphi^{\mathrm{ns}}$ & QHr/V ${ }^{\mathrm{ns}}$ & نرمال & & \\
\hline$r \mid N / \kappa^{\mathrm{ns}}$ & $\cdot /\left.q\right|^{\mathrm{ns}}$ & $1 / \Delta \gamma^{\mathrm{ns}}$ & $\cdot / v^{n s}$ & $\cdot / \cdot 1 r^{\mathrm{ns}}$ & $r \mid \Delta / \Delta^{\mathrm{ns}}$ & تنش & $\Delta$ & $\mathrm{Wr}-\mathrm{Vr}$ \\
\hline$r V / / 1$ & ऍ. & r r & T/ז & .1 .4 & rTD/V & نرمال & - & 1h- \\
\hline $19 N / r$ & $r / \mp \wedge$ &.$/ F \Delta$ & זسץ/. & $\% \cdots \Delta \Delta$ & r & 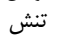 & & \\
\hline
\end{tabular}

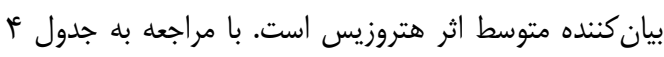

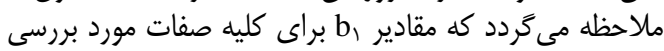

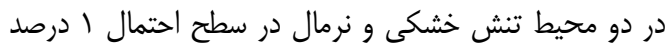

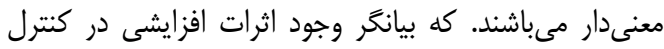

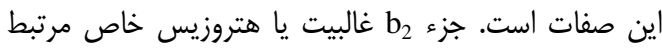

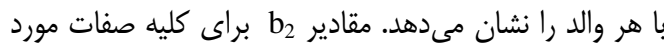

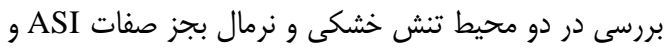

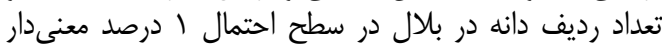

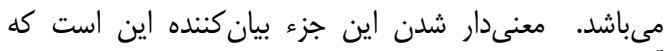

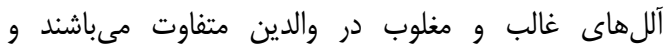

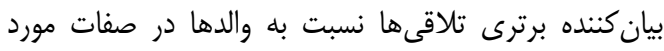

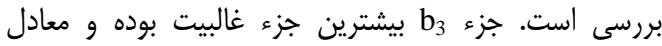

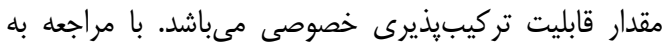

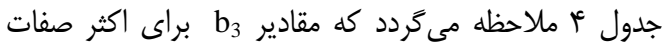

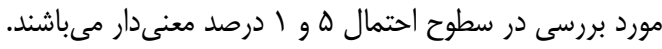

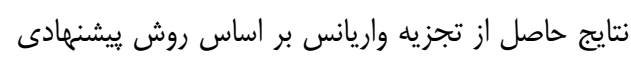

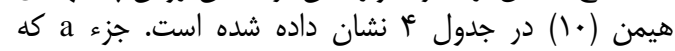

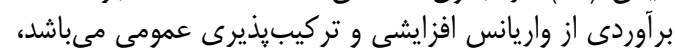

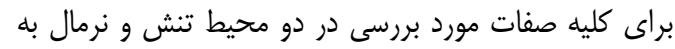

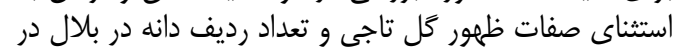

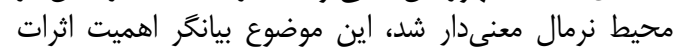

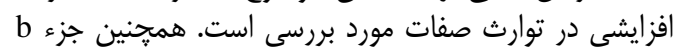

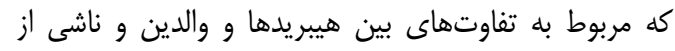

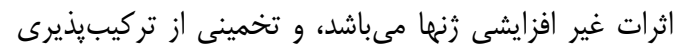

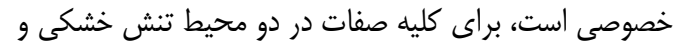

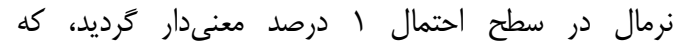

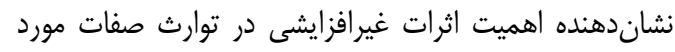

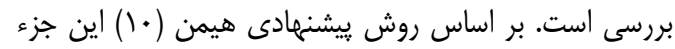

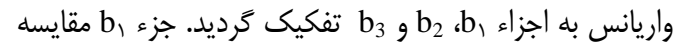
بين والدها در برابر تلاقىها مى باشد. به عبارت ديكر اين جزء 
جدول عأ- تفكيك ميانكين مربعات صفات مختلف ذرت به روش هيمن در دو شرايط نرمال و تنش خشكى Table 2. Analysis of variance of diallel Tables for various traits in maize under normal and water stress conditions

\begin{tabular}{|c|c|c|c|c|c|c|c|c|}
\hline دمر بوته (كرم) دانه & تعداد دانه در & تعداد رديفل & 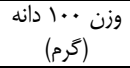 & ASI & (سانتى تبرت) & شرايط & آزادى & تنغيير \\
\hline $99 / V T^{6 / 2}$ & $q / / \mu^{*}$ & $f / / q^{*}$ & $9 / \kappa^{\prime \prime}$ & 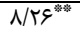 & $N / T \Lambda^{4 \cdot a}$ & نرمال & & \\
\hline$r r / v q^{*}$ & $\mid Q / \cdot \mu^{-3}$ & $F / \Lambda^{*}$ & $\Delta / r q^{* \prime \prime}$ & $T / 1 Q^{* * *}$ & $r+\varphi / r^{* * *}$ & تنش & $r$ & تكرار \\
\hline $\mid r \cdot 1 / T V^{*}$ & 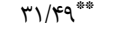 & 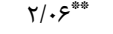 & $9 / 94^{* * a}$ & $1 / .4^{* \prime}$ & $\Delta \& 1 / 9 T^{*}$ & نرمال & & \\
\hline$V I M / r I^{*}$ & $r M / r e^{\text {絭 }}$ & $. / 9)^{* * 4 k}$ & 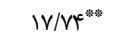 & $\cdot / r v^{*}$ & DIV/V **t* & تنش & r. & رنوتيّي \\
\hline TFT/MF": & $V / q . *$ & $1 /\left.4\right|^{*}$ & $\mid r / r$. *was & $1 / 98^{* * *}$ & $r T r / r \Lambda^{*}$ & نرمال & & \\
\hline $9 \mathrm{TV} / 99^{* * *}$ & $s / M^{*}$ & $\cdot / \Delta \mathrm{V}^{*}$ & $91 / \Delta V^{* * * a}$ & $\cdot \mid q^{* * *}$ & $1 \ldots / V V^{* * * a n}$ & تنش ت ك & ద & a \\
\hline$|Q T \cdot / 9|^{* a t}$ & $r q / r \Delta$ "w" & $T / T \Lambda^{\text {Natis }}$ & $91.9^{\text {w.w }}$ & $\cdot / N 8^{\| \prime \prime}$ & $g V G / r^{*}$ & نرمال & & \\
\hline 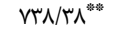 & $r \varepsilon / \cdot r^{\prime \prime \prime}$ & $1 / \cdot r^{* * *}$ & $r \cdot / 48^{* * * *}$ & $\cdot / T v^{* * *}$ & $9 \Delta S / 9 \mathrm{~N}^{* * 4}$ & تنش & 10 & b \\
\hline TrMEV/DI" & 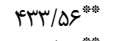 & 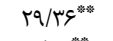 & $V Y / q r^{*: *}$ & $\Delta / V^{\mu *}$ & 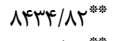 & نرمال & 1) & $b_{1}$ \\
\hline$q F \Delta \Lambda / \Delta \Lambda^{*}$ & rTr/V & $N / T \Lambda^{*}$ & 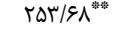 & $r / 4 \gamma^{*}$ & SHeq/Af & تنش & 1 & $\theta_{1}$ \\
\hline$r+q / Y \Lambda^{*}$ & $10 / 9 \Lambda^{*}$ & $1 / \cdot e^{\mathrm{ns}^{\prime}}$ & $r / \Delta r^{-w_{*}}$ & 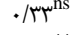 & $r \cdot V / \cdot r^{*}$ & نرمال & & \\
\hline 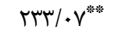 & 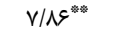 & $.190^{* \text { wath }}$ & $g / r)^{* \ldots+\infty}$ &.$/ r^{* * a n}$ & 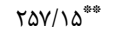 & تنش & 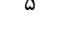 & $b_{2}$ \\
\hline$F+/ .1^{*}$ & $N / 9)^{*=6}$ & •/. rs & $.1 .9^{\mathrm{ns}}$ & $\cdot \mid q r^{\mathrm{ns}}$ & $V^{*} / \mathrm{Q}^{* * *}$ & نرمال & & \\
\hline$\Delta \cdot / \pi \cdot *$ & $r / \cdot \mathrm{e}^{\mathrm{ns}}$ &.$/ 4 \theta^{*}$ & $r / \epsilon^{* * *}$ & $\cdot 1 \cdot 0^{\mathrm{ns}}$ & Trq/. & تنش & 9 & $b_{3}$ \\
\hline $11 / . r$ & T/RT & .148 & .1 .1 & . / & $10 / r 9$ & ن نرمال & f. & \\
\hline$r T / \Lambda$. & $r / T$. & .119 & $1 / T \pi$ & .1 .4 & TL/Tr & تنش & $\Gamma^{\circ}$ & حطb \\
\hline$F / 9 \Lambda$ & G/AT & $\mathrm{V} / \mathrm{Vq}$ & $\Delta / V^{N}$ & $T r / K$. & $1 / M$ & نرمال & \multirow{2}{*}{\multicolumn{2}{|c|}{ ضريب تغييرات }} \\
\hline$\Delta / \Lambda T^{c}$ & $N / \pi \Delta$ & $1 . / \% \Lambda$ & $V / r q$ & $r q 1 \cdot 1$ & $r / r$ & تنش & & \\
\hline
\end{tabular}

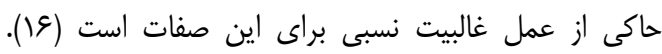

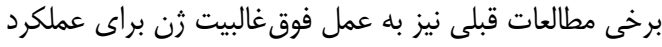

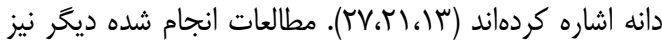

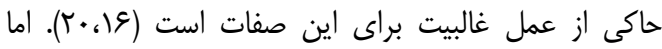

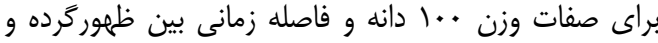

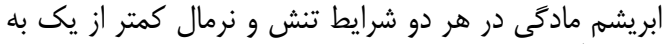

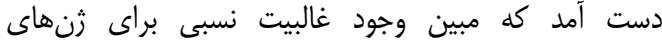

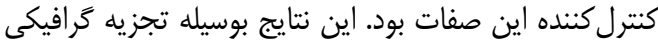

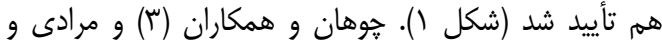

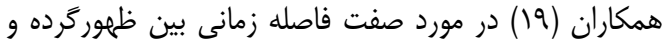

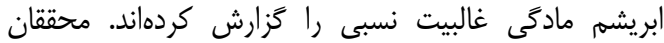

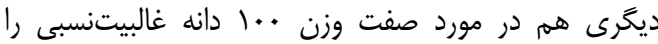

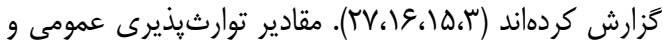

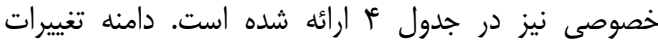

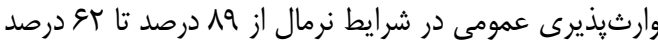

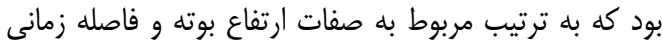

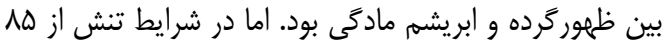

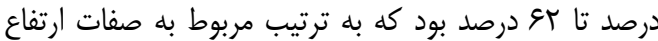

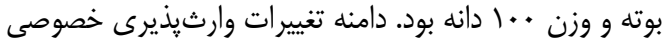

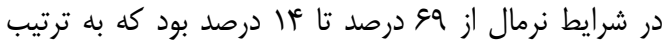

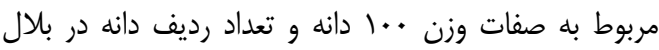

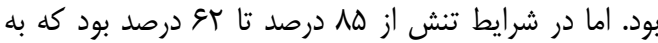

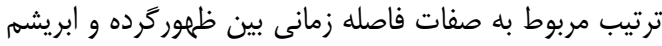

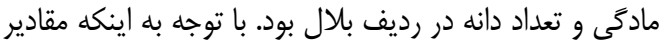

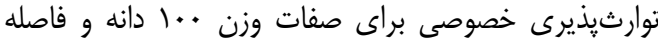

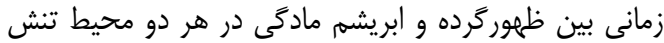

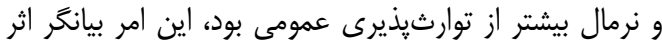

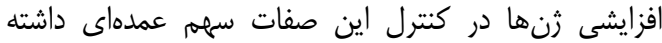

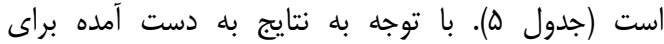

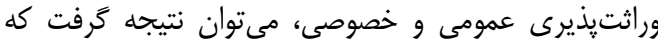
براى صفاتى كه وراثتيذيرى خصوصى بالايى دارند، انتخاب

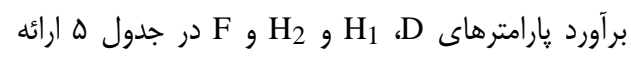

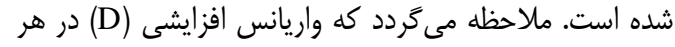

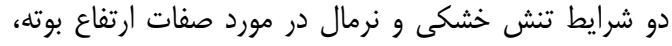

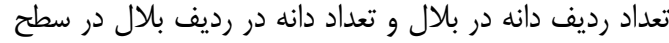

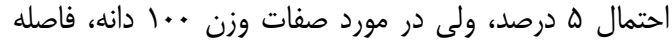

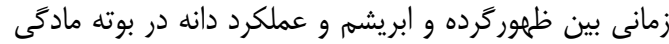

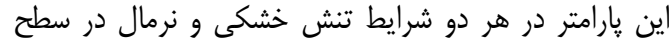

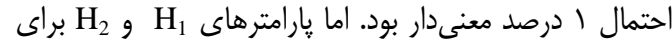

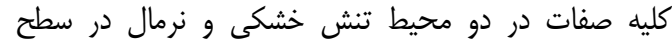

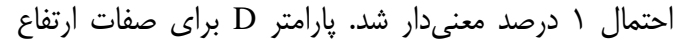

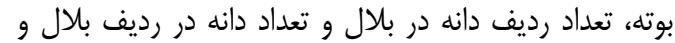

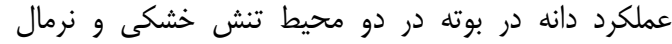
كو جكتر از يارامترهاى

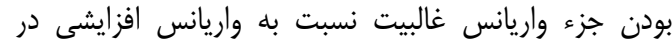

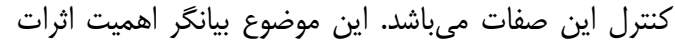

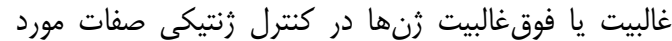

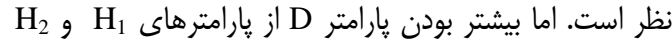

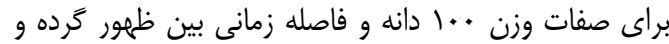

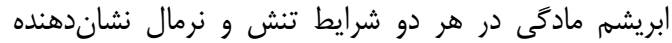

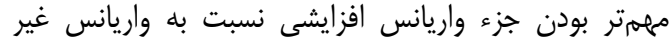

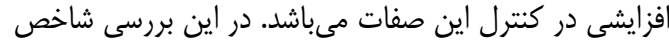

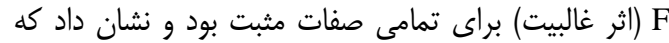

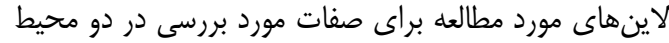

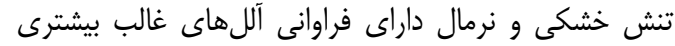

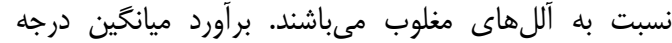

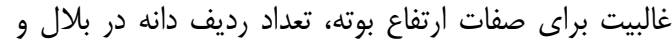

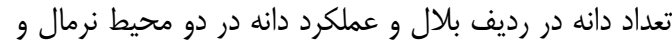

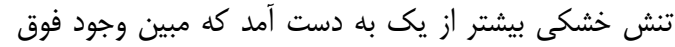

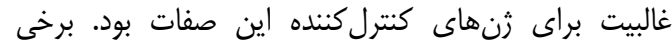

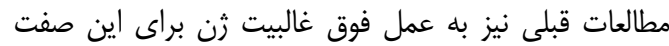

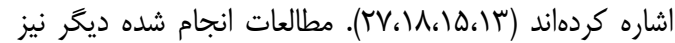


آزمايش باشد. آماره

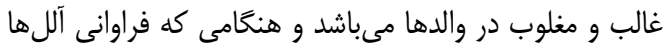

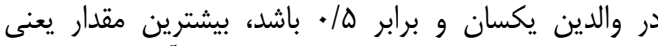

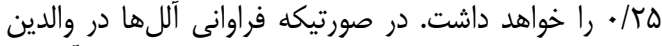

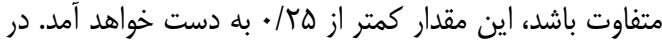

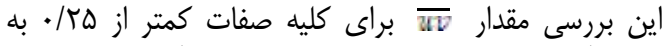

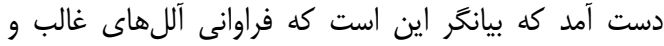
مغلوب در والدها يكسان نمىباشد.
در نسلهاى اوليه مىتواند موفقيت آميز باشد. يايين بودن

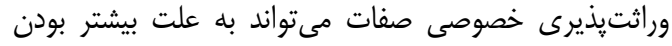
سهمه اثرات غيرافزايشى ثنها

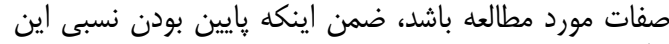

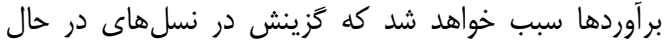

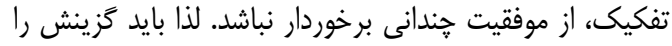

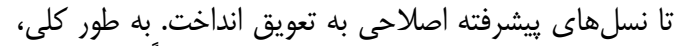

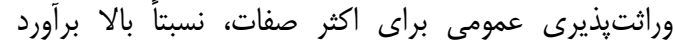
كرديد كه اين موضوع مىتواند ناشى از شرايط كنترل شده

جدول ه- بر آوردهاى يارامترهاى زنتيكى براى صفات مختلف ذرت در دو شرايط نرمال و تنش خشكى Table 5. Estimation of the components of genetic for various traits in maize under normal and water stress conditions

\begin{tabular}{|c|c|c|c|c|c|c|c|}
\hline در عملهرد دانه & تعداد دانه در & تعداد رديف & 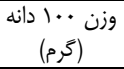 & ASI & (سانتىى بوتر) & شرايط & آماره \\
\hline$r W / c^{s / n}$ & $1 / A T^{*}$ & $\cdot / A F^{*}$ & $19 / 79$ & $\cdot / \Delta T^{* *}$ & $9 N / V 9^{\circ}$ & نرمال & \multirow{2}{*}{ واريانس افزايشى (D) } \\
\hline$r \cdot F / T$ & $1 / 91^{\circ}$ & " rו" & $r \mid / \Lambda)^{\cdots}$ & $\cdot / T \Lambda^{W \cdots}$ & $r F / \Delta \Lambda^{\circ}$ & تنش & \\
\hline $91 \Gamma / \kappa^{*}$ & $r \Delta / \Delta \Gamma^{* \tau}$ & $r / \cdot \mu^{*+}$ & $|V / F|^{* \pi}$ & $\cdot / 4 \varphi^{* \pi}$ & $\Delta V H / q^{* \pi}$ & 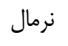 & \multirow{2}{*}{ واريانس غالبيت (H) } \\
\hline $9 \Lambda \varepsilon / 8$ & $r T / 1 Q$ &.$/ 19$ & $N / N^{\prime \prime}$ & " & $\Delta T r / \Delta$ & تنش & \\
\hline 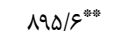 & MI/Fr" & $1 / \Delta r^{* *}$ & $1 F / 98^{*+}$ &.$/ 49^{* \pi}$ & $\Delta \mid N / F^{* \pi}$ & نرمال & \multirow{2}{*}{ واريانس غالبيت (H) } \\
\hline GTr/V & $r \cdot / \Delta \Delta^{-\cdots}$ & $\cdot / V D^{-W}$ & $\mid V / \cdot r^{w "}$ &.$/ 19^{\cdots+}$ & FAT/V & تنش & \\
\hline $\mid f V / T^{w}$ & $r / \Delta T$ & $\cdot / 0$ & 1." & $\cdot / M$ & $9 Q / \bar{s}^{\circ}$ & 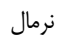 & \multirow{2}{*}{$\mathrm{F}$} \\
\hline$r \cdot r / V$ & $1 / 19$ & או/. & $\mu / \cdot f$ & זו/. & $1 / \Delta \mid$ & تنش & \\
\hline r/\%q & . /AT & $\cdot / \cdot V$ & . & זו/. & $\Delta / \Gamma \Lambda$ & نرمال & \multirow{2}{*}{ واريانس محيطى (E) } \\
\hline $\mathrm{V} / \mathrm{M}$ &.$/ 19$ & .1 .9 & • & 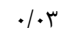 & $9 / \cdot \varphi^{\circ}$ & تنش & \\
\hline$r / T r$ & $F / F T$ & $r / \kappa \Delta$ & $\cdot|\Lambda|$ & r & $r / 19$ & نرمال & \multirow{2}{*}{ متوسط درجه غالبيت } \\
\hline l/Ar & $r / A f$ & $r / \Delta q$ & •/VD &.$/ 19$ & $r / 91$ & تنش & \\
\hline$\cdot|9|$ &.$|8|$ & $\cdot \mid \& \Delta$ & $\cdot / \Delta \Lambda$ & س און & $\cdot / \Delta \Lambda$ & 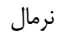 & \multirow{2}{*}{ نسبت ثنهاى غالب } \\
\hline س ז/. & .101 & .19 & $\cdot / 4$ & .198 & $\cdot|\Delta|$ & تنش & \\
\hline$\cdot / K F$ & $\cdot / \pi r$ & . & $\cdot|r|$ & $\cdot / K F$ & r & 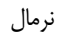 & \multirow{2}{*}{$\overline{U V}$} \\
\hline ז/א. & ז/א. & $\cdot|r|$ & . $/ 4 T$ & $\cdot|r|$ & . & تنش & \\
\hline .191 & - /AT & $\cdot / \mathrm{V} \Lambda$ & .190 & . & $\cdot / 19$ & نرمال & \multirow{3}{*}{ قابليت توارث عمومى } \\
\hline .180 & $\cdot|\wedge|$ & $\cdot / V T$ & . Iat & $\cdot / V T$ & - /AD & تنش & \\
\hline זא/. &.$/ 1 f$ &.$/ 1 f$ &.$/ 99$ &. $\mid 9 V$ & $\cdot / 1 \Lambda$ & نرمال & \\
\hline$\cdot / T V$ &.$/ 19$ &.$/ T r$ & - /Va & $\cdot / V \&$ & $\cdot / K \Lambda$ & تنش & \multirow{3}{*}{ قابليت توارث خصوصى } \\
\hline $1 f V / V \pi$ & $r \Delta / V T$ & $1 \cdot|9|$ & (T//QQ & $\Delta / \Gamma \mu$ & $11 \cdot / 4$ & نرمال & \\
\hline$|\Gamma \&| \wedge \Delta$ & $r r / \Lambda q$ & १/१९ & $r \cdot / 91$ & T/Tr & $\mid s+/ 1 V$ & تنش & \\
\hline $1 \wedge q / 4 F$ & $M / \Delta F$ & $I T / N$ & TV/TY & D/q ( D & $r \cdot \Delta / 99$ & 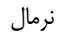 & \multirow{2}{*}{ ميانكين نتاج } \\
\hline$|\Delta r / V|$ & TV/AV & $1 . / \mathrm{VG}$ & $r \Delta / T^{c}$ & r/Va & $\mid \Lambda \varepsilon /$ q $^{\prime}$ & تنش & \\
\hline.$/ 48$ & . $/$ / &.$/ 94$ & ./94 &.$/ 9 V$ & . & 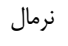 & \multirow{2}{*}{ ضريب ركر سيون } \\
\hline .199 & . $/ \Lambda \mu$ & $\cdot / \mathrm{VA}$ & .119 &.$/ 9 V$ & $\cdot \mid \& \Delta$ & تنش & \\
\hline
\end{tabular}

نشاندهنده عمل غالبيت نسبى و فوق غالبيت زنها منا مياشد.

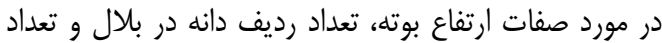

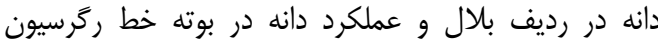

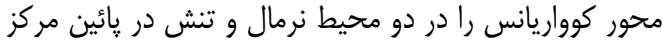

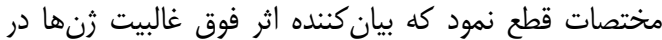

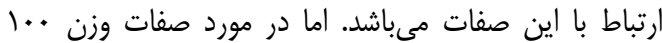

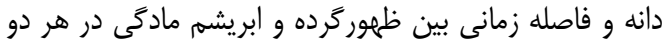

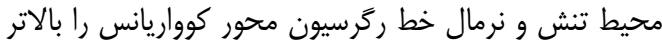

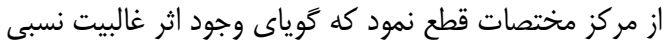
ثنها در كنترل اين صفات مىباشد.
شكلهاى الف تا ظ سهمى هاى محدودكننده و خطوط

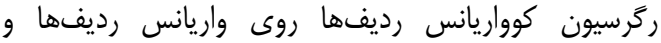

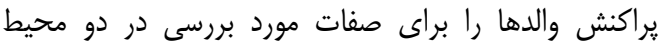

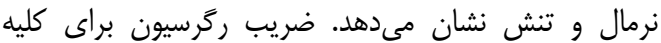

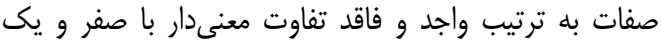

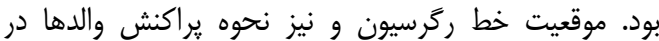

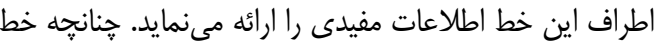

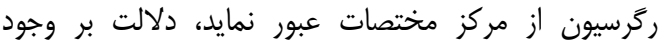

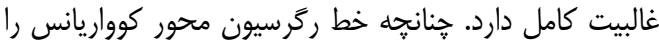
در بالا يا در ڤائين مركز مختصات قطع كند به به ترتيب 

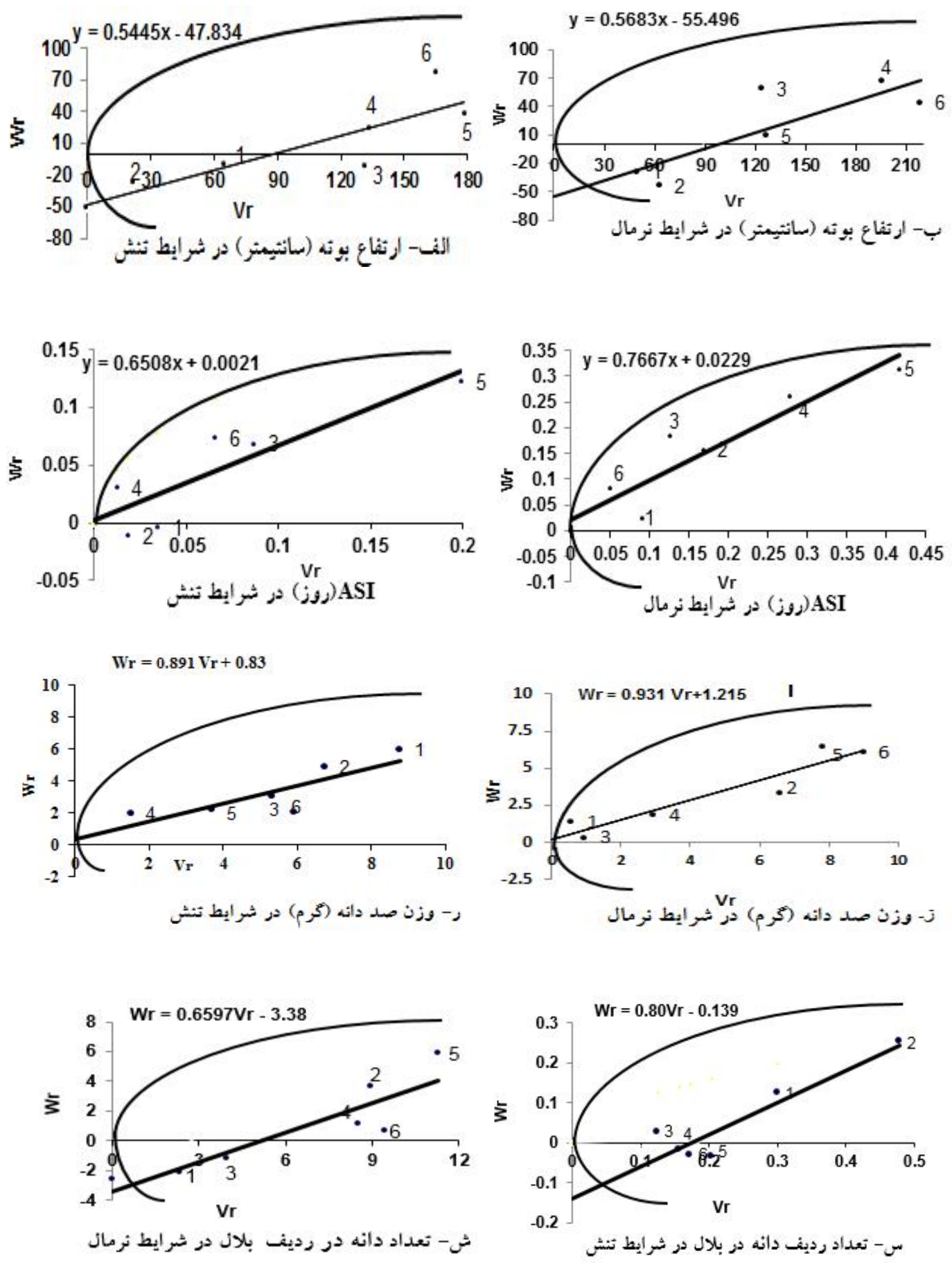

1: CML, 2: SD13, 3: SD\17, 4: SD\10, 5: SD\15 and 6: SD।704

شكل ا- تجزيه تحليل كرافيكى عملكرد و صفات وابسته براى تلاقىهاى داى آلل در دو شرايط نرمال و تنش خشكى

Figure 1. Vr/Wr graphs analysis for grain yield related traits using diallel crosses under normal and water stress conditions 

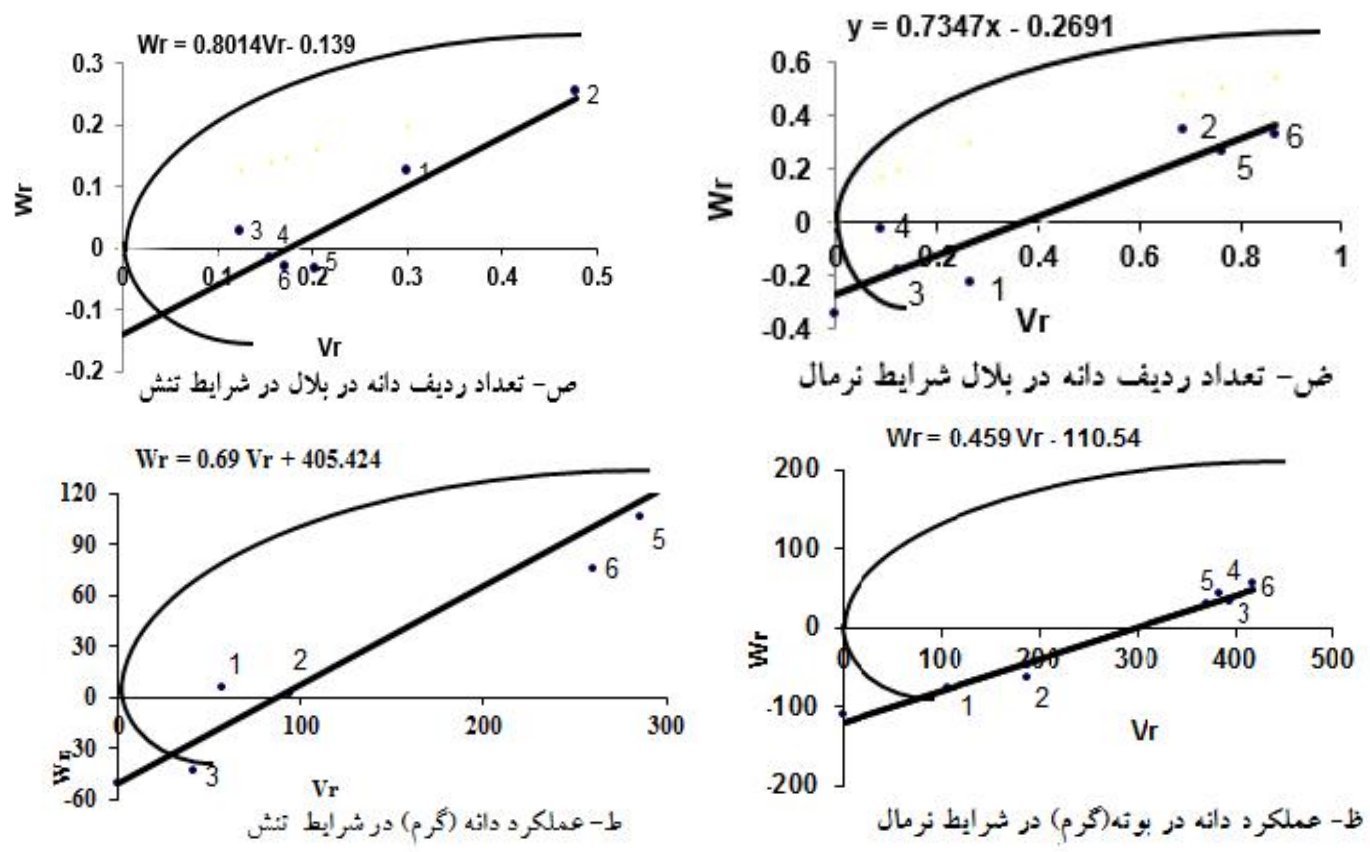

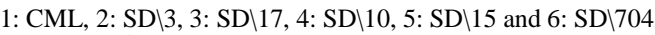

شكل (- تجزيه تحليل كرافيكى عملكرد و صفات وابسته براى تلاقى هاى داى آلل در دو شرايط نرمال و تنش خشكى

Figure 1. Vr/Wr graphs analysis for grain yield related traits using diallel crosses under normal and water stress conditions

تريبريدهاى توليدى كمك شايان توجهى در دستيابى به مانه

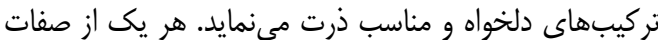

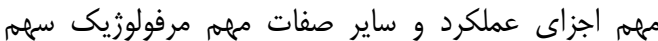

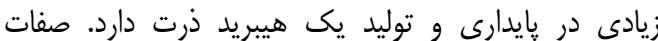

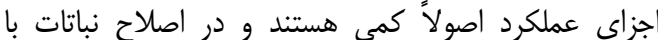

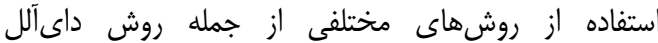

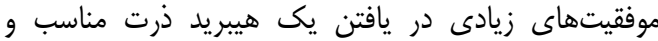

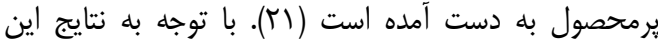

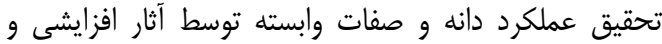

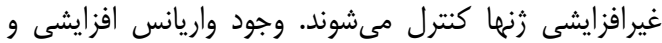

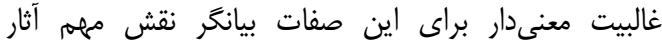

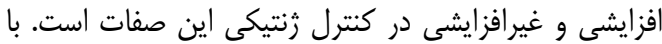

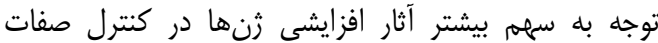

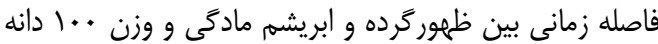

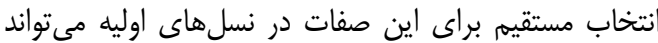

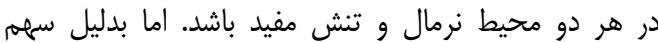

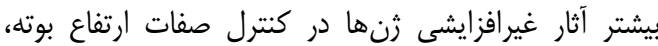

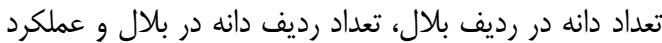

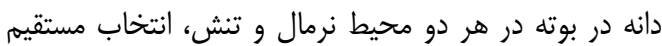

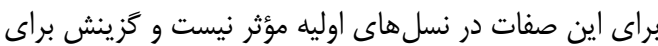

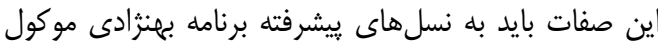

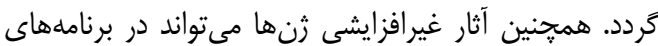

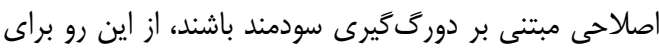

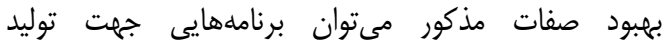
زرنوتيبهاى دورى تدارك ديد.
يراكنش والدها در اطراف خط رگرسيون بيانخر فراوانى

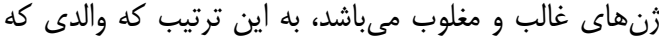

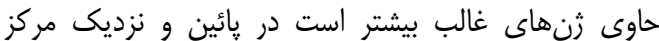

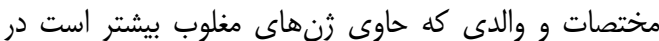

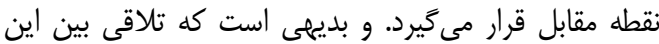

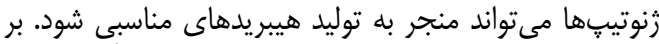

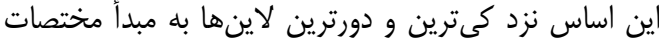

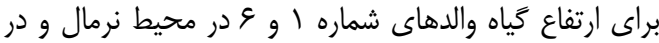

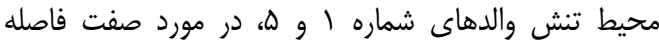

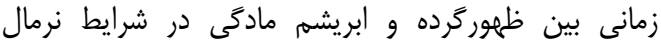

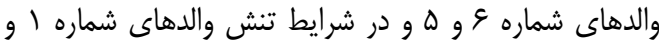

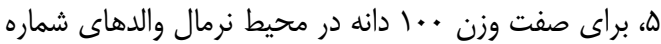

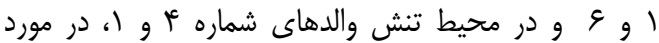

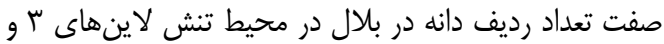

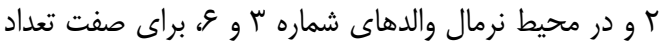

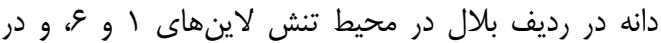

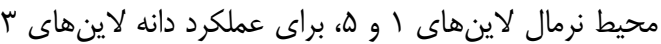

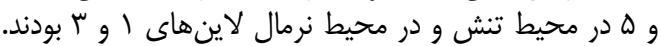

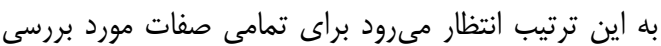

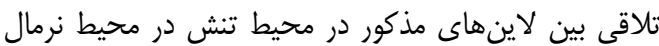

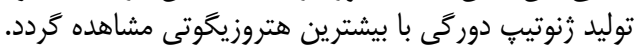

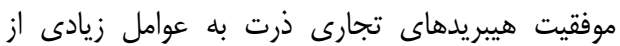

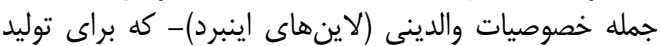

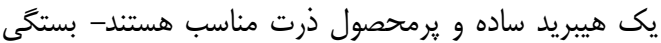
دارد. شناخت والدين هيبريد در ذرت و ارتباط بين بين والدين و 
1. Andjelkovic, V., D. Ignjatovic-Micic, S. Mladenovic and J. Vancetovic. 2012. Implementation of maize gentic resources in drought tolerance and grain quality improvement at maize research institute."Zemun Polje".Thiyrd International Scientific Smposium UDK 631, 147: 63-75.

2. Betran, F.J., J.M. Ribaut, D. Beck and D.G. Leon. 2003. Genetic diversity, specific combining ability and heterosis in tropical maize under stress and non-stress environments. Crop Science, 43: 797-806.

3. Chohan, M.S.M., M. Saleem, M. Ahsan and M. Asghar. 2012. Genetic analysis of water stress tolerance and various morpho-physiological traits in (Zea mays L.) using graphical approach. Pakistan Journal of Nutrition, 11: 489-500.

4. Chowdhry, A.M., M. Rafing and K. Alam. 1992. Genetic architecture of grain yield and certain other traits in breed wheat. Pakistan Journal Agricultural Science, 13: 216-220.

5. Emam, I. and M. Nicnejad. 1994. An introduction on physiological of crop yield. Shiraz University press. Shiraz. Iran. $240 \mathrm{pp}$ (In Persian).

6. Farshadfar, E. 1996. Application of biometrical genetics in plant breeding. Razi University press. Kermanshah. Iran. 198 pp (In Persian).

7. FAO. 2010. Statistical data. www. FAOSTAT. Org.

8. Griffing, B. 1956. Concept of general and specific combining ability in relation to diallel crossing systems. Australian Journal of biological science, 9: 463-493.

9. Gardner, C.O. and S.A. Eberhart. 1966. Analysis and interpretation of the varity cross diallel and related populations. Biometery, 22: 439-459.

10. Hayman, B.I. 1957. Interaction, heterosis and diallel crosses. Genetics, 42: 33-35.

11. Hayman, B.I. 1963. Notes on diallel cross theory, in: Statistical genetics and plant breeding. NASNRC 982:5 71-578.

12. Hayman, B.I. 1958. The theory and analysis of diallel crosses. Genetics, 32: 789-809.

13. Hussain, M., K.N. Shah., A. Ghafoor., T.T. Kiani and T. Mahmood. 2014. Genetic analysisfor grain yield and various morfological traits in corn (Zea mays L.) under normal and water stress environmets. The Journal of Animal and Plant Sciences, 24: 1230-1240.

14. Imtiaz, H., A. Muhammad, S. Muhammad and A. Ashfagh. 2009. Gene action studies for agronomic traits in maize under normal and water stress conditions. Pakistan Journal Agricultural Science, 46: 107-113.

15. Irshad-Ul-Haq, M., S. Ullah Ajmal, M. Munir and M. Gulfaraz. 2010. Gene action studies of different quantitative traits in maize (Zea mays L.). Pakistan Journal Botany, 42: 1021-1030.

16. Jinks, J.L. 1954. The analysis of continouse variation in a diallel crosses of nicotiana rustica varieties. Genetics, 39: 67 -78.

17. Jinks, J.L. and B.I. Hayman. 1953. The analysis of diallel crosses. Maize Genetics Cooperation Newsletter, 27: 48-54.

18. Mather, K. and J.L. Jinks. 1982. Biometrical genetics. Chapman and Hall: London, 276 pp.

19. Moradi, M., R. Choukan, E. Majidi Heravan and M.R. Bihamta. 2014. Genetic analysis of various morpho-physiological traits in (Zea mays L.) using graphical approach under normal and water stress conditions. Research on Crops, 15: 62-70.

20. Mostafave, K., R. Choukan, E. Majidi Heravan, M.R. Bihamta and M. Taeb. 2007. Genetic control studies of different traits in maize inbred lines (Zea mays L.) using graphical analysis. Seed and Plant Improvment Journal, 4: 117-129 (In Persian).

21. Mostafave, K., R. Choukan, E. Majidi Heravan, M. R, Bihamta and M. Taeb. 2009. Genetic studies of grain yield and related traits in corn (Zea mays L) using graphical analysis Journal of Agronomy and Plant Breeding, 6:117-129 (In Persian).

22. Sadeghi, F. and J. Rotbeh. 2016. Evaluation of grain yield and yield components using descriptive and multivariate statistics. Journal of Crop Breeding, 8: 211-221 (In Persian).

23. Sadeghi F. and Rotbeh J. 2016. The use of cluster analysis for best lines selection in maize at S6 generation, Journal of Crop Breeding, 8: 91-98 (In Persian).

24. Sridic, J., S.S. Mladenoivic-Drinic and Z. Pajic. 2006. Combining abilities and genetic resemblance of maize lines. Acta Agronomica Humgarica, 54: 337-342.

25. Von Braun, J., D. Byerlee, C. Charters, T. Lumpkin, N. Olembo and J. Waage. 2010. A draft strategy and results framework for the CGIAR. The world bank, Washington DC. $312 \mathrm{pp}$.

26. Walter, D.E. and J.R. Morton. 1978. On the analysis of variance of half diallel table. Biometery, 34: 91-94.

27. Wattoo, F.M. and M. Saleem. 2009. Genetic analysis for yield potential and Quality Traits in of maize (Zea mays L.). American- Eurasian Journal Agriculture. And Environment Science, 6: 723-729.

28. Yadav, O.P. and S.K. Bathagar. 2001. Evaluation of indices for identification of pear millet cultivars adapted to stress and non-stress conditions. Field crop Research, 70: 201-208. 


\title{
Graphical Analysis for Grain Yield Related Traits in Maize (Zea mays L.) using Diallel Crosses under Normal and Water Stress Conditions
}

\author{
Mohammad Moradi ${ }^{1}$ and Rajab Choukan ${ }^{2}$ \\ 1- Assistant Professor, Department of Plant Breeding, shoushtar Branch., Islamic Azad University, Shoushtar, Iran \\ (Corresponding author: email: moradim17@ yahoo.com) \\ 2- Profssors, Seed and Plant Improvement Institute, Karaj, Iran. \\ Received: June 5, 2016 \\ Accepted: February 4, 2016
}

\begin{abstract}
This study was carried out in order to investigate the genetic structure of the $F_{1}$ hybrids along with their parents ( 6 inbred lines) evaluated in field under normal and water stress regimes to determine the nature and magnitude of genetic variances and heritability estimates at dezful research station in safi abad, from 2012, using RCBD with three replications. Results analysis of variance showed that Variation among genotypes were highly significant for all the traits studied under both regimes. Therefore, variations were partitioned into additive and non additive components according to Hayman and Hayman \& Jinks methods. It was observed that $\mathrm{a}$ and $\mathrm{b}$ components which are due to additive and dominant gene effects respectively, were significant for all studied characteristics under both conditions. Considering the average degree of dominance, as well as $\mathrm{Vr} / \mathrm{Wr}$ graphs revealed that the yield potential traits like plant height, grain number in row in ear, grain row number in ear, and grain yield per plant were controlled by over dominance type of gene action, while traits like ASI, and 100-kernel weight were under the control of partial dominance with additive type of gene action under both conditions. Overdominance for most of the parameters reveals that selection in later generations may be more effective and the selection in early generations will be more effective for the trait which is additively controlled.
\end{abstract}

Keywords: Drought stress, Genetic parameters, Graphic plot, Maize 